\title{
Relação entre variáveis ambientais e a estrutura da comunidade de peixes em córregos das bacias do Rio das Mortes e do rio Xingu - MT, Brasil
}

\author{
Priscylla Rodrigues Matos * \\ Carolina Mancini do Carmo \\ Cesar Enrique de Melo \\ Universidade do Estado de Mato Grosso, Laboratório de Ictiologia e Limnologia \\ Caixa Postal 08, CEP 78690-000, Nova Xavantina - MT, Brasil \\ * Autor para correspondência \\ matosprm@hotmail.com
}

Submetido em 29/11/2012

Aceito para publicação em 19/06/2013

\section{Resumo}

As variáveis ambientais podem determinar e estruturar a composição da fauna de peixes. Estudos que comparem diferenças entre variáveis físicas e químicas da água entre bacias hidrográficas próximas são escassos. Este artigo teve por objetivo verificar quais variáveis limnológicas estão relacionadas à distribuição das espécies de peixes em duas bacias hidrográficas. Para tanto, foram amostrados 20 córregos, divididos entre as bacias do Rio das Mortes e do rio Xingu. Em cada ponto foram medidos um total de 8 variáveis ambientais. Os peixes foram coletados com rede de arrasto. A riqueza total foi de 57 espécies, sendo 29 da bacia do rio Xingu, 35 do Rio das Mortes e 7 espécies comuns a ambas as bacias. As análises demonstraram que os córregos dessas duas bacias têm características limnológicas e fauna distintas. Os córregos da bacia do rio Xingu tiveram menores valores de $\mathrm{pH}$ que podem ter sido influenciados pelas elevadas taxas de decomposição orgânica. Os córregos do Rio das Mortes apresentaram valores mais elevados de matéria em suspensão e clorofila, provavelmente ocasionadas pela maior degradação dos córregos e menores níveis de cobertura vegetal.

Palavras-chave: Amazônia; Cerrado; Riacho

\section{Abstract}

Relation between environmental variables and the fish community structure in streams of das Mortes and Xingu river basins - MT, Brazil. Environmental variables may determine and structure the composition of fish fauna. Studies comparing differences between physical and chemical variables of water between close river basins are few. This paper aimed to check which limnological variables are related to the distribution of fish species in two river basins. For this, 20 streams were sampled, divided between das Mortes and Xingu river basins. At each point one measured a total of 8 environmental variables. Fishes were collected through trawl. Total richness was 57 species, 29 of them from Xingu river basin, 35 from das Mortes river basin, and 7 species common to both river basins. The analyses showed that the streams in these two basins have distinct limnological and faunal features. The streams in Xingu river basin had lower $\mathrm{pH}$ values which may have been influenced by the high rates of organic decomposition. The streams of das Mortes river showed higher values of suspended matter and chlorophyll, probably due to higher degradation of streams and lower vegetation cover levels.

Key words: Amazon rainforest; Cerrado; Stream 


\section{Introdução}

As condições físicas e químicas de um ambiente aquático podem determinar e estruturar a composição da fauna de peixes. Entre as variáveis físicas podemos citar: declividade, profundidade, velocidade da água, turbidez, sólido totais dissolvidos, condutividade, temperatura da água, substrato do leito, tamanho da bacia e cobertura vegetal (RINCÓN, 1999; UIEDA; CASTRO, 1999; ALLAN; CASTILLO, 2007; ARÁUJO; TEJERINAGARRO, 2007; 2009; VALÉRIO et al., 2007; TUNDISI; TUNDISI, 2008; WINEMILLER, et al., 2008; MELO et al., 2009). Entre as variáveis químicas destacam-se: pH, oxigênio dissolvido, nitrogênio total e orgânico (RINCÓN, 1999; TUNDISI; TUNDISI, 2008; ARAÚJO; TEJERINA-GARRO, 2009; ESTEVES, 2011).

As bacias do Rio das Mortes e do rio Xingu, embora próximas, possuem características geomorfológicas distintas (LOWE-MCCONNELL, 1991; VALENTE; LATRUBESSE, 2009; SILVA et al., 2013). Essas diferenças influenciam as características ambientais e irão gerar diferentes condições ecológicas, o que pode influenciar a estrutura da ictiofauna (LOWEMCCONNELL, 1991; VALENTE; LATRUBESSE, 2009; SILVA et al., 2013). No entanto, nem todas as variáveis podem explicar a estrutura da comunidade de peixes. Araújo e Tejerina-Garro (2009) demonstraram que de dez variáveis ambientais mensuradas apenas quatro, velocidade da água, largura do canal, temperatura da água e $\mathrm{pH}$, foram estruturadoras das assembleias de peixes. Mudanças temporais ou estruturais na bacia de drenagem podem fazer com que algumas variáveis, que em alguns locais são importantes para a distribuição das espécies de peixes, como pH e oxigênio dissolvido, não sejam relevantes para esse mesmo grupo de organismos em outros locais (VALÉRIO et al., 2007).

Portanto, se as características físicas e químicas de um ambiente aquático influenciam na estrutura da comunidade de peixes, torna-se fundamental compreender quais dessas variáveis ambientais são importantes localmente, para que, este estudo possa servir de material auxiliar em estratégias de conservação, já que este possui informações importantes sobre a ecologia aquática de ambientes tão pouco estudados.
Dessa forma esse trabalho teve como objetivo verificar quais variáveis físicas e químicas estão relacionadas com a abundância, riqueza e diversidade de espécies de peixes em pequenos córregos nas bacias do rio Xingu e do Rio das Mortes, com o intuito de responder às seguintes questões: 1) As condições físicas e químicas da água e do ambiente variam entre as bacias hidrográficas analisadas? 2) Existem variáveis físicas e químicas que estão relacionadas com a distribuição das espécies de peixes nos córregos? 3) Existe correlação significativa entre tamanho das micro-bacias e riqueza de espécies, abundância de indivíduos e/ou diversidade?

\section{Material e Métodos}

\section{Área de estudo}

Foram amostrados 20 córregos, 10 córregos pertencentes à bacia hidrográfica do rio Xingu, que está inserida na Bacia Amazônica (Figura 1), no município de Querência e 10 córregos na bacia do Rio das Mortes, que está inserida na Bacia Araguaia-Tocantins (Figura 1), no município de Nova Xavantina, ambos no estado de Mato Grosso.

Nos afluentes da bacia do Rio das Mortes a vegetação predominante foi o cerrado stricto sensu. Para essa mesma região, de acordo com a classificação de Köppen o clima é tropical $(A w)$ com duas estações bem definidas (BRASIL, 1981). De acordo com dados da estação Meteorológica de Nova Xavantina, a temperatura média anual está entre 23,2 e $25,6^{\circ} \mathrm{C}$ com precipitação média anual de 1300 a 1600 mm, e maior intensidade de chuvas entre dezembro e fevereiro (LIMA, 2003). A maioria dos córregos estudados nesta bacia está parcialmente preservada ou degradada (Tabela 1), devido a transformação das APPs em pastagem para criação de gado bovino e a utilização dos córregos como bebedouros para esses animais (observação pessoal).

Os córregos afluentes do rio Xingu estão inseridos em área de floresta estacional perenifólia, a região é tropical chuvosa com temperatura média anual entre 24 e $26^{\circ} \mathrm{C}$ (BRASIL, 1981). A precipitação média anual é de 1900 a $2000 \mathrm{~mm}$, com maior intensidade de chuvas entre novembro e março (IPAM, Estação Meteorológica 
FIGURA 1: Localização dos pontos amostrais das bacias do rio Xingu (A) e Rio das Mortes (B).

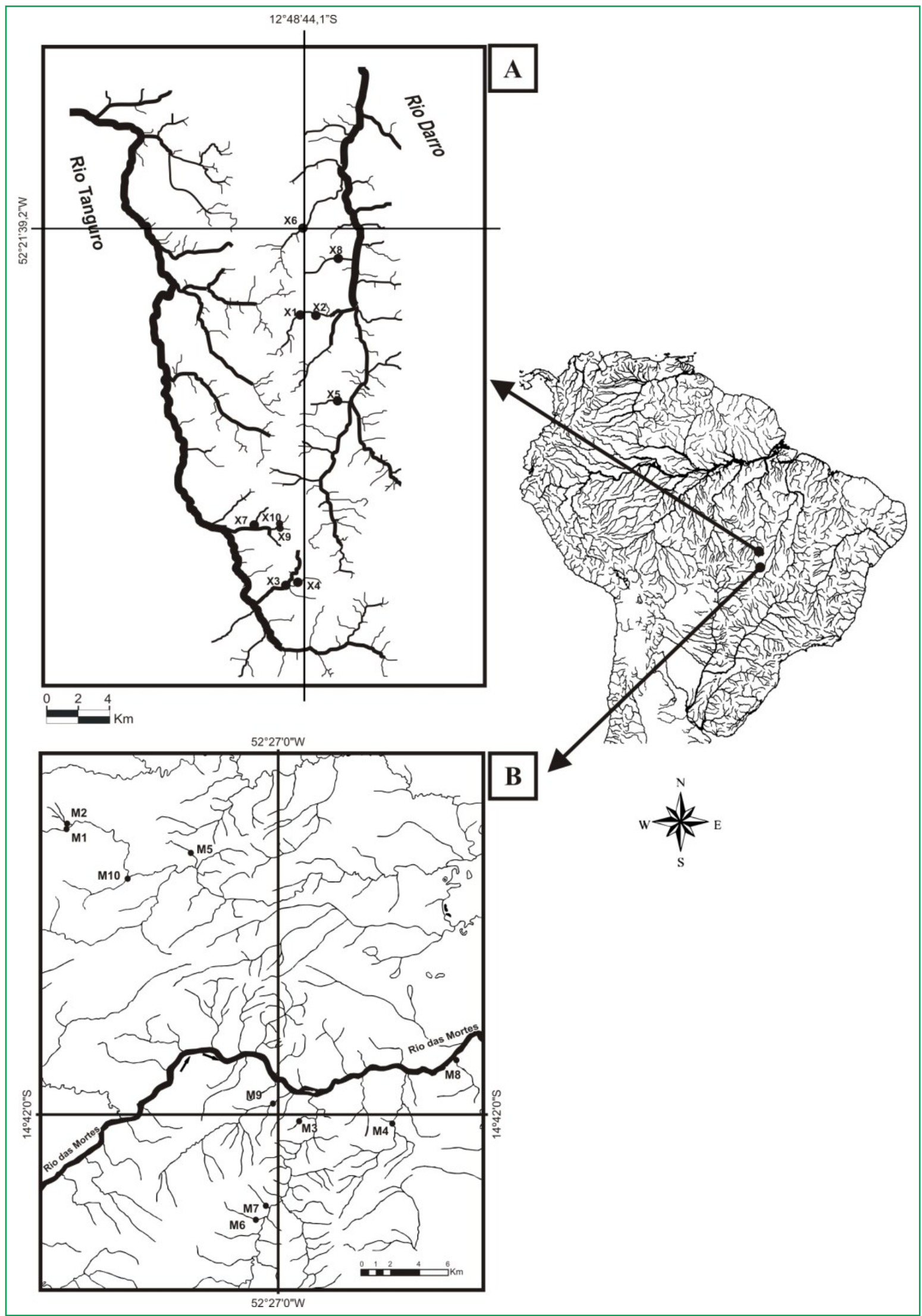


TABELA 1: Características dos córregos amostrados na bacia do rio Xingu (X) e do Rio das Mortes (M).

\begin{tabular}{|c|c|c|c|c|c|}
\hline Cór & Latitude $\mathrm{S}$ & Longitude W & Ordem & Substrato & Vegetação marginal \\
\hline $\mathrm{X} 1$ & $12^{\circ} 52^{\prime} 55,5^{\prime \prime}$ & $52^{\circ} 21^{\prime} 51,6^{\prime \prime}$ & 2 & Matéria orgânica & Preservada \\
\hline $\mathrm{X} 2$ & $12^{\circ} 52^{\prime} 55,5^{\prime \prime}$ & $52^{\circ} 21^{\prime} 01,8^{\prime \prime}$ & 2 & Areia & Preservada \\
\hline $\mathrm{X} 3$ & $13^{\circ} 06^{\prime} 10,1^{\prime \prime}$ & $52^{\circ} 22^{\prime} 34,5^{\prime \prime}$ & 2 & Areia & Preservada \\
\hline $\mathrm{X} 4$ & $13^{\circ} 05^{\prime} 58,9^{\prime \prime}$ & $52^{\circ} 22^{\prime} 02,8^{\prime \prime}$ & 1 & Matéria orgânica & Preservada \\
\hline $\mathrm{X} 5$ & $12^{\circ} 57^{\prime} 07,6^{\prime \prime}$ & $52^{\circ} 20^{\prime} 04,6^{\prime \prime}$ & 2 & Rocha e Areia & Parcialmente preservada \\
\hline $\mathrm{X} 6$ & $12^{\circ} 48^{\prime} 44,1^{\prime \prime}$ & $52^{\circ} 21^{\prime} 39,2^{\prime \prime}$ & 2 & Areia e Rocha & Ausência de vegetação \\
\hline $\mathrm{X} 7$ & $13^{\circ} 03^{\prime} 11,4^{\prime \prime}$ & $52^{\circ} 24^{\prime} 06,7^{\prime \prime}$ & 1 & Matéria orgânica & Parcialmente preservada \\
\hline $\mathrm{X} 8$ & $12^{\circ} 50^{\prime} 07,2^{\prime \prime}$ & $52^{\circ} 20^{\prime} 01,8^{\prime}$ & 2 & Areia fina e matéria orgânica & Preservada \\
\hline X9 & $13^{\circ} 03^{\prime} 20,5$ & $52^{\circ} 22^{\prime} 48,6^{\prime \prime}$ & 1 & Areia fina & Capim Brachiaria \\
\hline $\mathrm{X} 10$ & $13^{\circ} 03^{\prime} 8,6^{\prime \prime}$ & $52^{\circ} 22^{\prime} 47,6^{\prime \prime}$ & 1 & Matéria orgânica & Parcialmente preservada \\
\hline M1 & $14^{\circ} 30^{\prime} 39,0^{\prime \prime}$ & $52^{\circ} 35^{\prime} 11,4 ”$ & 2 & Matéria orgânica, Rocha & Preservada \\
\hline M2 & $14^{\circ} 30^{\prime} 32,3^{\prime \prime}$ & $52^{\circ} 35^{\prime} 18,4 ”$ & 2 & Matéria orgânica & Parcialmente preservada \\
\hline M3 & $14^{\circ} 42^{\prime} 16,4^{\prime \prime}$ & $52^{\circ} 26^{\prime} 03,4^{\prime \prime}$ & 1 & Rocha e Areia & Parcialmente preservada \\
\hline M4 & $14^{\circ} 42^{\prime} 19,5^{\prime \prime}$ & $52^{\circ} 22^{\prime} 25,1^{\prime \prime}$ & 3 & Rocha e Matéria orgânica & Parcialmente preservada \\
\hline M5 & $14^{\circ} 31^{\prime} 43,5^{\prime \prime}$ & $52^{\circ} 30^{\prime} 21,7^{\prime \prime}$ & 1 & Cascalho e Matéria orgânica & Degradada \\
\hline M6 & $14^{\circ} 46^{\prime} 02,6^{\prime \prime}$ & $52^{\circ} 27^{\prime} 52,0^{\prime \prime}$ & 1 & Cascalho e Areia & Parcialmente preservada \\
\hline M7 & $14^{\circ} 45^{\prime} 30,4^{\prime \prime}$ & $52^{\circ} 27^{\prime} 28,3^{\prime \prime}$ & 1 & Matéria orgânica e Cascalho & Preservada \\
\hline M8 & $14^{\circ} 39^{\prime} 53,2^{\prime \prime}$ & $52^{\circ} 19^{\prime} 51,8^{\prime \prime}$ & 2 & Rocha e Areia & Degradada, arbustos \\
\hline M9 & $14^{\circ} 41^{\prime} 32,7^{\prime \prime}$ & $52^{\circ} 27^{\prime} 08,3^{\prime \prime}$ & 3 & Matéria orgânica e Areia & Degradada \\
\hline M10 & $14^{\circ} 32^{\prime} 43,0^{\prime \prime}$ & $52^{\circ} 32^{\prime} 56,4 ”$ & 2 & Areia e Matéria orgânica & Parcialmente preservada \\
\hline
\end{tabular}

da Fazenda Tanguro). O clima da região e do tipo $A w$ de acordo com a classificação de Köppen (BRASIL, 1981). A maioria dos córregos amostrados apresenta APPs preservadas, com alguns ambientes de mata degradada (Tabela 1).

\section{Amostragem da ictiofauna}

Os peixes foram coletados no período de seca, entre o início de setembro e início de novembro de 2009 , período em que estes córregos apresentam melhores condições de trabalho e ausência da interferência do escoamento superficial. Para captura dos peixes foi utilizado rede de arrasto $(4,0 \mathrm{~m} \times 1,5 \mathrm{~m}$ e malha de 5 $\mathrm{mm})$, com tempo de coleta de 1 hora em cada ambiente, em um trecho de aproximadamente $200 \mathrm{~m}$. Os peixes coletados foram fixados em formol a $10 \%$ e, em seguida, transferidos para solução de álcool $70 \%$. Todo o material está tombado na coleção do Laboratório de Ictiologia e Limnologia da UNEMAT - Campus de Nova Xavantina.

\section{Variáveis ambientais}

As variáveis ambientais foram tomadas com equipamentos e metodologia específica, sendo que $\mathrm{pH}$, condutividade, oxigênio dissolvido na água, temperatura da água e do ambiente foram medidos com auxílio dos seguintes equipamentos digitais portáteis: peagômetro WTW mod. pH 315i), condutivímetro OAKTON mod. COM 6/TDS 6), oxímetro WTW mod. Oxi 315 i e termômetro digitais. A temperatura da água foi medida a $10 \mathrm{~cm}$ de profundidade em cada córrego. A temperatura do ambiente foi medida a 1 $\mathrm{m}$ do solo, na margem do ambiente que estava sendo amostrado. A turbidez foi determinada em laboratório, com água coletada nos locais de amostragem, com auxílio de turbidímetro digital de bancada ALFA TECNOQUÍMICA mod. SL 2K. A matéria em suspensão foi determinada pelo método de gravimetria por filtração a vácuo (PINTO-SILVA, 2002), também em laboratório. Para determinar a quantidade de 
clorofila "a", a água coletada em campo, em garrafa escura de dois litros, foi fixada com Carbonato de Magnésio saturado $\left(\mathrm{MgCO}_{3}\right)$ ainda em campo (PINTOSILVA, 2002). Em laboratório a amostra de água foi filtrada em filtros de membrana AP20 MILLIPORE. Estes filtros foram acondicionados em envelopes de papel alumínio e guardados em frascos escuros com sílica gel em freezer a $-18^{\circ} \mathrm{C}$ (PINTO-SILVA, 2002), posteriormente eles foram encaminhados, em caixa com gelo, para o laboratório do Instituto Internacional de Ecologia e Gerenciamento Ambiental - São Carlos, SP. Onde a clorofila "a" foi determinada pelo método tricromático.

\section{Análise dos dados}

Para averiguar se as variáveis físicas e químicas diferem entre bacias e influenciaram a distribuição das espécies de peixes foi realizada uma Análise de Correspondência Canônica (CCA) (NETO, 2011). As matrizes foram logaritmizadas para homogeneização dos valores das variáveis e não foi retirado o efeito das espécies raras, já que para peixes, as espécies raras fornecem informações específicas do local (POOS; JACKSON, 2012). Posteriormente foi realizado um teste de Monte Carlo com 500 permutações. Esse teste realiza randomizações para testar a significância dos eixos da CCA (LEGENDRE; LEGENDRE, 1998). O número de randomizações foi suficiente para estabilizar os valores de significância.

Para correlacionar o tamanho da área das microbacias de drenagem com a estrutura da comunidade de peixes, foi utilizada uma análise de regressão simples (ZAR, 1996; LEGENDRE; LEGENDRE, 1998; MAGURRAN, 2004), onde a estrutura da comunidade é a variável dependente e o tamanho da microbacia é a variável independente. A área das micro-bacias foi determinada com base em imagens de satélite. Para os pontos amostrais na bacia do rio Xingu, os dados foram fornecidos pelo IPAM e para os pontos amostrais da bacia do Rio das Mortes os dados foram cedidos pelo LANA-UNEMAT. A área de cada micro-bacia foi determinada como sendo a área de drenagem à montante de cada ponto de amostragem.
As diversidades de espécies e uniformidades foram calculadas pelo índice de Shannon-Wiener - H' (MAGURRAN, 2004) para cada córrego amostrado. A análise de regressão simples foi executada com auxílio do programa Statistica (Versão 7.0, StatSoft Inc.). A CCA e o teste de Monte Carlo foram realizados com o programa Canoco for Windows 4.5.

\section{Resultados}

Para os 20 pontos amostrais foram coletados 3.054 peixes, distribuídos em 57 espécies (Tabela 2). Nos 10 córregos da bacia hidrográfica do rio Xingu foram coletados 1.073 peixes de 29 espécies (Tabela 2), com variação entre quatro e 20 espécies por ambiente (Tabela 3). Nos córregos da bacia do Rio das Mortes foram coletados 1981 peixes, distribuídos em 35 espécies, com variação entre cinco e 20 espécies por córrego (Tabela 3). Apenas sete espécies foram comuns às duas bacias, são elas: Aequidens tetramerus, Bryconops giacopinii, Characidium gr. zebra, Chenicichla gr. labrina, Hemigramus cf. levis, Hoplias malabaricus e Rivulus zigonectes.

A análise de CCA ordenou as duas bacias hidrográficas em lados opostos do eixo 1 (Figura 2) e os dois primeiros eixos dessa análise explicaram $54,8 \%$ da ordenação (Tabela 4). As principais variáveis que explicaram a distinção entre os pontos amostrais das duas bacias hidrográficas e de suas respectivas ictiofaunas foram: $\mathrm{pH}$, matéria em suspensão e clorofila " $a$ " (Tabela 4) (Figura 2A e 2B). De forma geral, os córregos do Rio das Mortes apresentaram maiores valores médios de $\mathrm{pH}$, matéria em suspensão e clorofila " $a$ " (Tabela 5).

O teste de Monte Carlo aplicado aos eixos de ordenação da CCA demonstrou que a correlação entre as variáveis ambientais e as espécies envolvidas no estudo foi estatisticamente significativa para o eixo 1 $(\mathrm{F}=2,54 ; \mathrm{P}=0,002)$ como para o conjunto dos eixos da $\mathrm{CCA}(\mathrm{F}=1,299 ; \mathrm{P}=0,044)$. 
TABELA 2: Lista de espécies coletadas nos 20 pontos amostrais nas bacias hidrográficas: Rio das Mortes e rio Xingu com os códigos para cada espécie amostrada (Cód).

\begin{tabular}{|c|c|c|c|}
\hline ORDEM, Família e espécie & Cod. & Araguaia & Xingu \\
\hline \multicolumn{4}{|l|}{ CHARACIFORMES } \\
\hline \multicolumn{4}{|l|}{ Acestrorhynchidae } \\
\hline Acestrorhynchus falcatus (Bloch, 1974) & Ace & 2 & \\
\hline \multicolumn{4}{|l|}{ Characidae } \\
\hline Brycon pesu Müller \& Troschel, 1844 & Brp & 1 & \\
\hline Hemigrammus sp.3 & $\mathrm{He} 3$ & & 158 \\
\hline Hemigrammus sp.1 & Hem & & 42 \\
\hline Astyanax sp. & Ast & 1 & \\
\hline Creagrutus sp. & Cre & 5 & \\
\hline Bryconops cf giacopinii (Fernández-Yépez, 1950) & Bry & 36 & 1 \\
\hline Hemigrammus cf. levis Durbin, 1908 & Hel & 491 & 2 \\
\hline Knodus sp. & Kno & 844 & \\
\hline Moenkhausia oligolepis (Günther, 1864) & Moo & 162 & \\
\hline Astyanax novae Carl H. Eigenmann, 1911 & Asn & 20 & \\
\hline Moenkhausia pirauba Zanata, Birindelli \& Moreira, 2010 & Mop & 15 & \\
\hline Phenacogaster gr. pectinatus (Cope,1870) & Phe & 35 & \\
\hline Serrapinnus piaba (Lütken, 1875) & Sep & 160 & \\
\hline Jupiaba apenima Zanata, 1997 & Jap & 9 & \\
\hline Jupiaba polylepis (Günther, 1864) & Jpo & 17 & \\
\hline Moenkhausia collettii (Steindachner, (1882) & Moc & 11 & \\
\hline Astyanax elachylepis Bertaco \& Lucinda, 2005 & Ase & 1 & \\
\hline Moenkhausia phaeonota Fink, 1979 & Mop & & 156 \\
\hline Hemigrammus cf. ocellifer (Steindachner, 1882) & Heo & & 9 \\
\hline Bryconops sp.1 & $\mathrm{Br} 1$ & & 3 \\
\hline Moenkhausia sp.2 & Mo2 & & 13 \\
\hline Jupiaba anteroides (Géry, 1965) & Jup & & 9 \\
\hline Hemigrammus sp.2 & $\mathrm{He} 2$ & & 455 \\
\hline Metynnis cf. maculatus (Kner, 1858) & Mem & & 1 \\
\hline Astyanax sp.3 & As3 & 4 & \\
\hline Astyanax sp.1 & As1 & 23 & \\
\hline Astyanax sp.2 & As2 & 25 & \\
\hline \multicolumn{4}{|l|}{ Crenuchidae } \\
\hline Characidium gr. zebra Eigenmann, 1909 & $\mathrm{Chz}$ & 18 & 2 \\
\hline \multicolumn{4}{|l|}{ Curimatidae } \\
\hline Cyphocharax spiluropsis (Eigenmann \& Eigenmann, 1889) & Cyp & 5 & \\
\hline Steindachnerina amazonica (Steindachner, 1911) & Ste & 7 & \\
\hline \multicolumn{4}{|l|}{ Erythrinidae } \\
\hline Hoplias malabaricus (Bloch, 1794) & Hop & 10 & 8 \\
\hline \multicolumn{4}{|l|}{ Lebiasinidae } \\
\hline Pyrrhulina sp. & Pyr & & 19 \\
\hline \multicolumn{4}{|l|}{ Parodontidae } \\
\hline Parodon pongoensis (Allen, 1942) & Par & 1 & \\
\hline
\end{tabular}




\section{CYPRINODONTIFORMES}

Poeciliidae

Pamphoricthys cf. araguaiensis Costa, 1991

Pam

Rivulidae

Rivulus zygonectes Myers, 1927

Riv

1

\section{GYMNOTIFORMES}

Gymnotidae

Gymnotus sp.

Gym

Gymnotus cf. carapo Linnaeus, 1758

Gyc

2

Rhamphichthyidae

Gymnorhamphichthys sp.

Gyr

Sternopygidae

Eigenmannia cf. virescens (Valenciennes, 1836)

Eig

Sternopygus macrurus (Bloch \& Schneider, 1801)

Stm

\section{PERCIFORMES}

Cichlidae

Aequidens tetramerus (Heckel, 1840)

Aeq

$$
9
$$

44

Crenicichla gr. labrina (Spix \& Agassiz, 1831)

Cra

Lae

Laetacara araguaiae (Ottoni \& Costa, 2009)

Sat

Satanoperca jurupari (Heckel, 1840)

Rel

Retroculus cf. lapidifer (Castelnau, 1855)

$\mathrm{Cr} 1$

3

Crenicichla sp.1

\section{SILURIFORMES}

Callichthydae

Corydoras maculifer Nijssen \& Isbrücker, 1971

Aspidoras pauciradiatus Weitzman \& Nijssen, 1970

Cetopsidae

Helogenes marmoratus Günther, 1863

$\begin{array}{ll}\text { Com } & 2 \\ \text { Asp } & 6\end{array}$

Doradidae

*Doradidae juvenil

Heg

Heptapteridae

Rhamdia sp.

Dor

Rhamdia sp.1

Rha

4

1

Rh1

Loricariidae

Hisonotus sp.

His

Hemiancistrus spilomma Cardoso \& Luciana 2003

Hes

13

Hypostomus sp.

Hyp 34

Farlowella cf. oxyrryncha (Kner, 1853)

Far

1

1.981

* Individuo com apenas $1 \mathrm{~cm}$ de comprimento, devido a isso não foi possível identificar o gênero com segurança. 
FIGURA 2: A - Ordenação dos córregos pela Análise de Correspondência Canônica (CCA). Círculos escuros são os córregos da bacia do rio Xingu e os círculos claros são córregos da bacia do Rio das Mortes. B - Ordenação das espécies e características limnológicas na Análise de Correspondência Canônica (CCA).

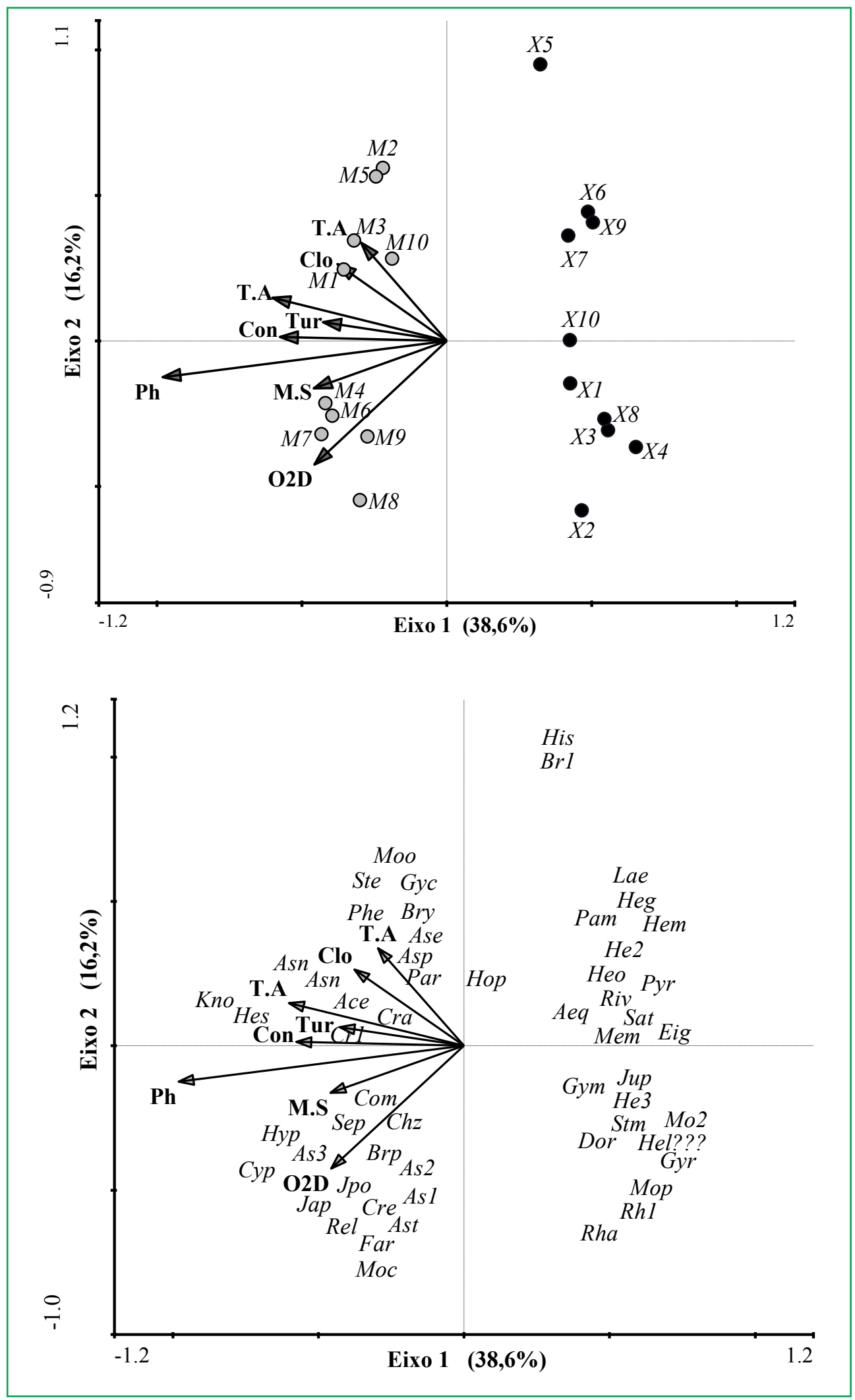


A mesma análise indicou que os ambientes com mata parcialmente preservada a degradada, representados pelos pontos amostrais: X5, X6, X7, X9 e X10 (Tabela 1), ficaram do lado oposto do mesmo eixo da CCA (Figura 2 A e B). Algumas espécies foram mais relacionadas a esses ambientes: Pamphoricthtys araguaiensis; Hemigrammus sp. 2, Sternopygus macrurus e Rivulus zigonectes. Já os córregos do rio Xingu com a mata ciliar preservada, representados pelos pontos amostrais: X1, X2, X3, X4 e X8 (Tabela 1), se posicionaram no lado negativo do eixo 2 (Figura 2 A e B). Esses ambientes apresentaram algumas espécies exclusivas ou com maior abundancia, como: Gymnorhamphichthys sp.; Moenkhausia phaeonota; Rhamdia sp. e Moenkhausia sp. 2.
TABELA 4: Auto-valores das variáveis limnológicas aferidas em cada córrego estudado, de acordo com os eixos da CCA, com a porcentagem da variância de cada eixo e a porcentagem acumulada de todos os eixos.

\begin{tabular}{lcc}
\hline \multicolumn{1}{c}{ Variáveis } & Eixo 1 & Eixo 2 \\
\hline $\mathrm{pH}$ & $\mathbf{- 3 , 0 1 1}$ & 0,008 \\
Condutividade & 0,029 & $-0,697$ \\
$\mathrm{O} 2 \mathrm{D}(\mathrm{mg} / \mathrm{L})$ & $-0,102$ & 0,241 \\
Temp. Ambiente $\left({ }^{\circ} \mathrm{C}\right)$ & 0,623 & $-0,065$ \\
Temp. da Água $\left({ }^{\circ} \mathrm{C}\right)$ & $-0,240$ & 0,619 \\
Turbidez $(\mathrm{NTU})$ & $-0,339$ & 0,676 \\
M. S. $(\mathrm{mg} / \mathrm{L})$ & $\mathbf{1 , 1 4 9}$ & $\mathbf{- 1 , 9 5 3}$ \\
Clorofila " $a$ " & $\mathbf{- 0 , 8 3 7}$ & $\mathbf{1 , 9 8 3}$ \\
\hline \% Variância Explicada & $\mathbf{3 8 , 6}$ & $\mathbf{1 6 , 2}$ \\
\%Variação Acumulada & $\mathbf{3 8 , 6}$ & $\mathbf{5 4 , 8}$ \\
\hline
\end{tabular}

TABELA 5: Variáveis limnológicas por córrego amostrado nas bacias hidrográficas do rio Xingu (X) e do Rio das Mortes (M), Mato Grosso, entre setembro e novembro de 2009.

\begin{tabular}{|c|c|c|c|c|c|c|c|c|}
\hline Córrego & $\mathbf{p H}$ & $\begin{array}{c}\text { Condutividade } \\
\left(\mu \mathrm{S} . \mathrm{cm}^{-1}\right) \\
\end{array}$ & $\begin{array}{c}\text { O2D } \\
\left(\mathrm{mg} . \mathrm{L}^{-1}\right)\end{array}$ & $\begin{array}{c}\text { Temp. } \\
\text { Ambiente } \\
\left({ }^{\circ} \mathrm{C}\right)\end{array}$ & $\begin{array}{c}\text { Temp. Água } \\
\left({ }^{\circ} \mathbf{C}\right)\end{array}$ & $\begin{array}{c}\text { Turbidez } \\
\text { NTU }\end{array}$ & $\begin{array}{c}\text { Mat. } \\
\text { Suspensão } \\
\left(\mathrm{mgL}^{-1}\right)\end{array}$ & $\begin{array}{c}\text { Clorofila "a" } \\
(\mu \mathrm{g} / \mathrm{L})\end{array}$ \\
\hline $\mathrm{X} 1$ & 5,04 & 6,50 & 3,99 & 25,60 & 23,80 & 4,92 & 0,001 & 1,89 \\
\hline $\mathrm{X} 2$ & 5,10 & 8,54 & 4,11 & 25,70 & 23,90 & 4,34 & 0,004 & 0,62 \\
\hline $\mathrm{X} 3$ & 4,95 & 6,72 & 6,49 & 27,30 & 24,40 & 0,41 & 0,001 & 1,13 \\
\hline $\mathrm{X} 4$ & 4,81 & 7,46 & 5,23 & 27,90 & 24,30 & 0,26 & 0,003 & 1,38 \\
\hline $\mathrm{X} 5$ & 5,25 & 4,81 & 6,00 & 30,80 & 27,90 & 3,55 & 0,002 & 2,59 \\
\hline X6 & 5,09 & 4,48 & 7,35 & 30,90 & 26,50 & 0,48 & 0,000 & 1,98 \\
\hline $\mathrm{X} 7$ & 5,24 & 4,62 & 4,94 & 30,60 & 28,50 & 1,26 & 0,002 & 1,03 \\
\hline X8 & 5,10 & 6,54 & 5,88 & 29,70 & 24,30 & 3,90 & 0,001 & 1,02 \\
\hline $\mathrm{X} 9$ & 5,01 & 8,15 & 4,85 & 30,20 & 26,60 & 0,74 & 0,001 & 2,46 \\
\hline $\mathrm{X} 10$ & 5,08 & 6,34 & 4,38 & 26,80 & 25,80 & 4,75 & 0,002 & 1,12 \\
\hline M1 & 6,63 & 13,77 & 7,14 & 28,20 & 25,50 & 3,43 & 0,003 & 1,75 \\
\hline M2 & 6,20 & 13,11 & 5,42 & 27,40 & 25,90 & 5,95 & 0,004 & 2,60 \\
\hline M3 & 6,68 & 62,30 & 5,93 & 32,90 & 26,90 & 15,47 & 0,004 & 1,18 \\
\hline M4 & 7,39 & 108,00 & 6,18 & 34,10 & 25,80 & 23,51 & 0,050 & 9,93 \\
\hline M5 & 6,68 & 84,80 & 3,52 & 35,50 & 27,30 & 4,03 & 0,006 & 6,41 \\
\hline M6 & 7,03 & 7,79 & 7,65 & 30,50 & 24,80 & 0,00 & 0,001 & 0,91 \\
\hline M7 & 7,28 & 10,85 & 7,43 & 33,20 & 24,70 & 1,13 & 0,001 & 0,82 \\
\hline M8 & 7,04 & 67,60 & 6,68 & 33,20 & 27,70 & 4,51 & 0,006 & 1,47 \\
\hline M9 & 6,76 & 6,65 & 7,22 & 28,40 & 25,90 & 3,55 & 0,005 & 1,53 \\
\hline M10 & 6,54 & 22,09 & 6,03 & 31,60 & 25,40 & 9,81 & 0,008 & 3,56 \\
\hline Médias - X & 5,06 & 6,41 & 5,32 & 28,55 & 25,6 & 2,46 & 0,002 & 1,52 \\
\hline Médias - M & 6,82 & 39,7 & 6,32 & 31,5 & 26 & 7,14 & 0,008 & 3,01 \\
\hline
\end{tabular}


As regressões não revelaram resultados estatisticamente significativos para as relações entre o tamanho da área à montante do ponto amostral de cada microbacia de drenagem e a estrutura da ictiofauna, composta por riqueza, abundância, diversidade e uniformidade (Tabela 6)

TABELA 6: Resultados da análise de regressão para as relações entre área da micro-bacia de drenagem e Riqueza, Abundância, Diversidade e Uniformidade da ictiofauna, com valores de significância (p).

\begin{tabular}{cccc}
\hline Área (ha) VS & $\mathbf{r}^{2}$ & $\mathbf{R}$ & $\mathbf{P}$ \\
\hline Riqueza & 0,004 & $-0,065$ & 0,787 \\
Abundância & 0,004 & $-0,063$ & 0,792 \\
Diversidade & 0,013 & $-0,112$ & 0,637 \\
Uniformidade & 0,000 & 0,925 & 0,916 \\
\hline
\end{tabular}

\section{Discussão}

Variáveis ambientais como $\mathrm{pH}$, matéria em suspensão, clorofila " $a$ ", turbidez e condutividade estão diretamente associadas com as estruturas dos córregos e níveis de degradações (TUNDISI; TUNDISI, 2008; ESTEVES, 2011). Os menores valores de $\mathrm{pH}$ dos córregos do rio Xingu estão associados a maior densidade florestal nessa região. A intensa decomposição da massa vegetal, devido à mata ciliar mais densa nos pontos amostrais da bacia do rio Xingu, acarreta a infiltração de ácidos húmicos para o lençol freático, que determinam aumento nos processos de acidificação das águas nos córregos dessa bacia (BRANDÃO; LIMA, 2002; ESTEVES, 2011).

A interferência do $\mathrm{pH}$ sobre a composição das comunidades de peixes é relatada em vários trabalhos (ABES; AGOSTINHO, 2001; TUNDISI; TUNDISI, 2008; FIALHO et al., 2008; ARAÚJO; TEJERINAGARRO, 2009). A grande abundância de Rivulus zygonectes e Aequidens sp. nos córregos da bacia do rio Xingu, onde as águas são mais ácidas, e sua raridade nos córregos da bacia do Rio das Mortes, sugere que essas variáveis ambientais influenciam na distribuição destas espécies nos pontos de coleta.
A substituição da vegetação nativa por agroecossistemas altera vários parâmetros limnológicos, entre estes, a condutividade elétrica, devido ao aumento de nutrientes e concentração de sólidos suspensos provenientes da lixiviação de áreas cultivadas (CARVALHO et al., 2000). Nos córregos da bacia do Rio das Mortes é evidente a maior condutividade, onde também ocorrem os mais intensos processos de degradação da mata de galeria, ocupada principalmente por pastagens. Processo este, muito mais intenso do que o observado na bacia do rio Xingu, que de forma geral, apresenta as matas de galeria mais preservadas.

O aumento de matéria em suspensão na água pode ser interpretado como evidência de degradação ambiental (RIOS; CALIJURI, 1995; CARVALHO et al., 2000; GORDON et al., 2004). Essa ocorrência é muito comum no estado de Mato Grosso, principalmente em áreas de pastagens onde o gado bovino utiliza os córregos como bebedouros. Nos córregos estudados na bacia do Rio das Mortes o pisoteio dos animais nas margens e leitos dos córregos provoca erosão e aumenta a entrada de sedimento nos cursos d'água (CARVALHO et al., 2000). Enquanto na bacia do rio Xingu esse processo não foi verificado nos pontos de amostragem.

Como o aumento de matéria em suspensão determina o aumento da turbidez, esse processo pode afetar severamente a composição da fauna de peixes, já que com a água mais turva os peixes que dependem da visão para se orientar podem ser suprimidos (MELO et al., 2009) ou apresentar redução em sua abundância. De forma geral, em águas mais turvas, aumenta a ocorrência de espécies de peixes que possuem estruturas secundárias, como barbilhões táteis, que auxiliam no processo de orientação em ambientes de menor luminosidade (MELO et al., 2005; KAVALCO; PAZZA, 2007) Isso explicaria a maior abundância de peixes da ordem Siluriformes nos córregos amostrados na bacia do Rio das Mortes em relação aos córregos do rio Xingu.

A maior quantidade média de clorofila " $a$ " detectada nos córregos afluentes do Rio das Mortes pode estar associada a dois fatores principais, ambos associados a diminuição da mata de galeria desses córregos: primeiro, aumento da luminosidade na água (SIMPAÚBA-TAVARES et al., 1995; WEBSTER et 
al., 1995;) e segundo, maior entrada de nutrientes originários das pastagens, os quais, em conjunto podem determinar um acentuado acréscimo da produtividade primária em ambientes aquáticos (BASSOLI; ROLAND, 2005; DAVIES et al., 2008; TUNDISI; TUNDISI, 2008; ARAÚJO; TEJERINA-GARRO, 2009; ESTEVES, 2011) além disso, a menor acidez das água com pH médio próximo ao neutro diminuem o stress aumentando a produtividade das comunidades fitoplanctônicas (BIUDES; CAMARGO, 2008). Esse aumento na produção primária pode determinar o estabelecimento de algumas espécies de peixes que utilizam diretamente essas algas ou os detritos originário dessas como recurso alimentar (DAVIES et al., 2008; TUNDISI; TUNDISI, 2008), como algumas espécies que foram encontradas apenas nos córregos da bacia do Rio das Mortes: Steindachnerina amazonica, Cyphocharax spiluropsis, Hemiancistrus spilomma, Hypostomus sp. e Farlowella cf. oxyrryncha, todas detritívoras ou algívoras (HOEINGHAUS et al., 2003; MELO et al., 2004). Essas guildas tróficas não foram encontradas nos pontos amostrados nos córregos da bacia do Xingu, que apresentaram menor produtividade primária.

Entre os córregos da bacia do rio Xingu, aqueles que apresentam matas ciliares mais alteradas, inseridos em áreas com pastagens ou soja, como os pontos amostrais: X5, X6, X7, X9 e X10, apresentaram condutividades mais baixas e temperaturas da água mais elevadas. Dois fatores podem estar determinando essas condições: em primeiro lugar a menor proporção de cobertura vegetal nesses pontos, que aumenta a incidência de luz no ambiente é consequentemente a temperatura da água (ARAÚJO; TEJERINA-GARRO, 2009); em segundo, a ocorrência de reservatórios à montante dos pontos de amostragens em todos os córregos de áreas alteradas, esses reservatórios podem reter nutrientes e diminuir a concentração dos mesmos à jusante da barragem, diminuindo a condutividade elétrica da água nos locais de coletas.

A CCA ainda indica que algumas espécies de peixes estão mais relacionadas a esse tipo de ambiente: Pamphoricthtys araguaiensis; Hemigrammus sp. 2, Sternopygus macrurus e Rivulus zigonectes, sendo que as três primeiras espécies são exclusivas dos ambientes com alterações na mata.

Os córregos do interior da floresta, inclusos em áreas cujas matas cillares estão preservadas, representados pelos pontos amostrais X1, X2, X3, X4 e $\mathrm{X} 8$, ao contrário do primeiro grupo em áreas alteradas, apresentaram maiores condutividades médias e menores temperaturas médias da água, entre os ambientes do rio Xingu, fatores que podem ser influenciados pela presença da mata de galeria, que promove maior sombreamento, diminuindo a temperatura e lançando material alóctone no ambiente, fonte de íons, que pode levar ao aumento da condutividade (CLETO-FILHO; WALKER, 2001; TUNDISI; TUNDISI, 2008; ESTEVES, 2011). A esse grupo de pontos amostrais estão mais relacionadas as seguintes espécies: Gymnorhamphichthys sp., Moenkhausia phaeonota, Rhamdia sp. e Moenkhausia sp. 2. Dessas, as três primeiras espécies foram encontradas apenas nos córregos com matas íntegras.

Embora o tamanho de uma bacia hidrográfica possa ser relacionado com o volume de água e o número de micro-hábitats disponíveis nos ambientes aquáticos (GORDON et al., 2004), a relação entre esta variável e o número de espécies de peixes ainda não está bem explicada. Miranda e Mazzoni (2003) verificaram que ocorre um aumento da riqueza de espécies, à medida que a bacia de drenagem à montante aumenta. No entanto Pazin (2004) demonstra que há dificuldades em relacionar tamanho de áreas com aumento de diversidade, riqueza ou abundância de peixes. A estrutura da comunidade parece estar mais associada com outras características físicas do ambiente como profundidade, velocidade da água, largura, comprimento ou volume do trecho amostrado (ANJOS; ZUANON, 2007; ARAÚJO; TEJERINA-GARRO, 2007). Neste estudo não houve relação significativa entre o tamanho das áreas de drenagem à montante dos pontos amostrais e a estrutura das comunidades de peixes nesses locais.

Assim, os pontos amostrais inclusos nas bacias hidrográficas do Rio das Mortes e do rio Xingu, apresentam variáveis físicas e químicas diferentes, influenciadas provavelmente pela geologia, diferentes tipos de vegetação e uso do solo. As principais variáveis que distinguem os córregos amostrados nas duas 
bacias são $\mathrm{pH}$, condutividade, clorofila “a', matéria em suspensão e turbidez. Essas variáveis limnológicas também estão associadas com a estrutura da ictiofauna nos ambiente estudados.

\section{Agradecimentos}

Agradecemos ao IPAM e ao Grupo André Maggi pelo apoio da estrutura física na Fazenda Tanguro bacia do rio Xingu. Aos alunos do Laboratório de Ictiologia e Limnologia da UNEMAT-Campus de Nova Xavantina e ao Sr Jovercino Rodrigues da Silva pelas preciosas ajudas de campo. Este trabalho recebeu auxilio financeiro da FAPEMAT, CNPq e CAPES/PROCAD.

\section{Referências}

ABES, S. S.; AGOSTINHO, A. A. Spatial patterns in fish distributions and structure of the ichthyocenosis in the Água Nanci stream, upper Paraná River basin, Brazil. Hydrobiologia, Brussels, v. 445, p. 217-227, 2001.

ALLAN, J. D.; CASTILLO, M. M. Stream ecology: structure and function of running waters. 2 ed. Dordrecht: Springer, 2007. 436 p.

ANJOS, M. B.; ZUANON J. Sampling effort and fish species richness in small terra firme forest streams of central Amazonia, Brazil. Neotropical Ichthyology, Porto Alegre, v. 5, n. 1, p. 45-52, 2007.

ARAÚJO, N. B.; TEJERINA-GARRO, F. L. Composição e diversidade da ictiofauna em riachos do Cerrado, bacia do ribeirão Ouvidor, alto rio Paraná, Goiás, Brasil. Revista Brasileira de Zoologia, Curitiba, v. 24, n. 4, p. 981-990, 2007.

ARAÚJO, N. B.; TEJERINA-GARRO, F. L. Influence of environmental variables and anthropogenic perturbations on stream fish assemblages, Upper Paraná River, Central Brazil. Neotropical Ichthyology, Porto Alegre, v. 7, n. 1, p. 31-38, 2009.

BASSOLI, F.; ROLAND, F. Caminhos da produção fitoplanctônica em ecossistemas aquáticos continentais. In: ROLAND, F.; CESAR, D.; MARINHO, M. (Ed.). Lições de limnologia. São Carlos: Rima, 2005. p. 173-191.

BIUDES, J. F. V.; CAMARGO, A. F. M. Estudos dos fatores limitantes à produtividade primária por macrófitas aquáticas no Brasil. Oecologia Brasiliensis, Rio de Janeiro, v. 12, n. 1, p. 7-19, 2008.

BRANDÃO, S. L.; LIMA, S. C. pH e condutividade elétrica em solução do solo, em áreas de pinus e cerrado na chapada, em Uberlândia (MG). Caminhos de Geografia, Uberlândia, v. 3, n. 6, p. 46-56, 2002.

BRASIL - MINISTÉRIO DAS MINAS E ENERGIAS. SECRETARIA GERAL. Projeto Radambrasil - Folha SD 22. Goiás: Geologia, Geomorfologia, Pedologia, Vegetação, Uso Potencial de Terra. Brasília: MME, 1981. 640 p.
CARVALHO, A. R.; SCHLITTLER, F. H. M.; TORNISIELO, V. L. Relações da atividade agropecuária com parâmetros físicos químicos da água. Química Nova, São Paulo, v. 23, n. 5, p. 618$622,2000$.

CLETO FILHO, S. A. N; WALKER, I. Efeitos da ocupação urbana sobre a macrofauna de invertebrados aquáticos de um igarapé da cidade de Manaus/AM - Amazônia Central. Acta Amazonica, Manaus, v. 31, n.1, p. 69-89, 2001.

DAVIES, P. M.; BUNN, W. E.; HAMILTON, S. K. Primary production in tropical. In: DUDGEON, D. (Ed.). Tropical stream ecology. 1 ed. Amsterdam: Academic Press, 2008. p. 24-43.

ESTEVES, F. A. Fundamentos de Limnologia. 3. ed. Rio de Janeiro: Interciência, 2011. 790 p.

FIALHO, A. P.; OLIVEIRA, L. G.; TEJERINA-GARRO, F. L.; MÉRONA, B. Fish-habitat relationship in a tropical river under anthropogenic influences. Hydrobiologia, Brussels, v. 598 p. $315-$ $324,2008$.

GORDON, D. N.; MCMAHON, T. A.; FINLAYSON, B. L.; GIPPEL, C. J.; NATHAN, R. J. Stream hydrology: an introduction for ecologists. 2. ed. New York: John Wiley \& Sons Ltd, 2004. 526 p. HOEINGHAUS, D. J.; LAYMAN, C. A.; ARRINGTON, D. A.; WINEMILLER, K O. Spatiotemporal variation in fish assemblage structure in tropical floodplain creeks. Environmental Biology of Fishes, Dordrecht, v. 67, p. 379-387, 2003.

KAVALCO, K. F.; PAZZA, R. Aspectos biogeográficos de componentes da ictiofauna da América Central. ConScientiae Saúde, São Paulo, v. 6, n. 1, p. 147-153, 2007.

LEGENDRE, P.; LEGENDRE, L. Numerical ecology. Amsterdam: Elsevier, 1998. 853 p.

LIMA, J. D. Diversidade, estrutura trófica da ictiofauna e condições limnológicas em um lago na planície inundável do Rio das Mortes - Mato Grosso - Brasil. 2003. 110 f. Dissertação (Mestrado em Ecologia e conservação) - Universidade Federal do Mato Grosso, Cuiabá. 2003

LOWE-MCCONNELL, E. H. Natural history of fishes in Araguaia and Xingu Amazonia tributaries, Serra do Roncador, Mato Grosso Brasil. Icthyological Exploration Freshwaters, München, v. 2, n. 1, p. 63-82, 1991 .

MAGURRAN, A. E. Measuring biological diversity. Oxford: Blackwell Science, 2004. 256 p.

MELO, C. E.; MACHADO, F. A.; PINTO-SILVA, V. Feeding habits from a stream in the savanna of Central Brazil Araguaia Basil. Neotropical Ichthyology, Porto Alegre, v. 2, n. 1, p. 37-44, 2004.

MELO, C. E.; LIMA, J. D.; MELO, T. L.; PINTO-SILVA, V. Peixes do Rio das Mortes identificação e ecologia das espécies mais comuns. Cuiabá: Unemat, 2005. 147 p.

MELO, C. E.; LIMA, J. D.; SILVA, E. F. Relationships between water transparency and abundance of Cynodontidae species in the Bananal floodplain, Mato Grosso, Brazil. Neotropical Ichthyology, Porto Alegre, v. 7, n. 2, p. 251-256, 2009.

MIRANDA, J. C.; MAZZONI, R.. Composição da ictiofauna de três riachos do Alto Rio Tocantins - GO. Biota Neotropica, São José do Rio Preto, v. 3, n. 1, p. 1-11, 2003.

NETO, S. C. S. Interação entre ambiente e espécies no interior e na borda de um fragmento de floresta estacional semidecidual. 2011. 
69. Dissertação (Mestrado em Ciências Florestais) - Universidade Federal do Espírito Santo, Jerônimo Monteiro. 2011.

PAZIN, V. F. V. Assembléias de peixes em poças temporárias marginais riachos de terra-firme, Amazônia Central. 2004. 43 f. Dissertação (Mestrado em Ecologia) - Universidade Federal do Amazonas/INPA, Manaus. 2004.

PINTO-SILVA, V. Manual de análise limnológica: métodos e técnicas. Cuiabá: gráfica ufmt, 2002.95 p.

POOS, M. S.; JACKSON, D. A. Addresing the removal of rare species in multivariate bioassessments: The impact of methodological choices. Ecological Indicators, Kiel, v18, p. 8290, 2012

RINCÓN, P. A. Uso do micro-hábitat em peixes de riachos: métodos e perspectiva. In: CARAMASCHI, P. E.; MAZZONI, R.; PERESNETO, P. R. (Ed.). Ecologia de peixes de riachos. Vol. 6. Rio de Janeiro: PPGE-UFRJ, Série Oecologia Brasiliensis, 1999. p. 23-90.

RIOS, L; CALIJURI, M. C. A bacia hidrográfica do Ribeirão do Feijão: uma proposta de ordenação das sub-bacias através de variáveis limnológicas. Acta Limnologica Brasiliensia, Rio Claro, v. 7, p. 151-161, 1995.

SILVA, J. P.; PEREIRA, D. I.; AGUIAR, A. M.; RODRIGUES, C. Geodiversity assessment of the Xingu drainage basin. Journal of Maps, London, http://dx.doi.org/10.1080/17445647.2013.775085, 2013.

SIMPAÚBA-TAVARES, L. H.; LIGEIRO, S. R.; DURIGAN, J. G. Variação de alguns parâmetros limnológicos em um viveiro de piscicultura em função da luz. Acta Limnologica Brasiliensia, Rio Claro, v. 7, p. 138-150, 1995.
TUNDISI, J. G.; TUNDISI, T. M. Limnologia. São Paulo: Oficina de textos. 2008. $631 \mathrm{p}$.

UIEDA, V. S.; CASTRO, R. M. C. Coleta e fixação de peixes de riachos. In: CARAMASCHI, P. E.; MAZZONI, R.; PERES-NETO, P. R. (Ed.). Ecologia de peixes de riachos. Vol. 6. Rio de Janeiro: PPGE-UFRJ, Série Oecologia Brasiliensis, 1999. p. 1-22.

VALENTE, C. R.; LATRUBESSE, E. M. Regional geomorphologic mapping of the Araguaia River Basin, Central Brazil. In: XIV Simpósio Brasileiro de Sensoriamento Remoto, 4921, 2009, Natal. Resumo. Natal: INPE, 2009. Versão eletrônica.

VALÉRIO, S. B.; SÚAREZ, Y. R.; FELIPE, T. R. A.; TONDATO K. K.; XIMENES, L. Q. L. Organization patterns of headwaterstream fish communities in the Upper Paraguay-Paraná basins. Hydrobiologia, Brussels, v. 583, p. 241-250, 2007.

WEBSTER, J. R.; WALLACE, J. B.; BENFIELD, E. F. Organic processes in streams of eastern United States. In: ALLAN, J. D.; CASTILLO, M. M. (Ed.). Stream ecology structure and function of running waters. 2. ed. Dordrecht: Springer, 1995. p. 117-187.

WINEMILlER, K. O.; AGOSTINHO, A. A.; CARAMASCHI, É. P. Fish ecology in tropical streams. In: DUDGEON, D. (Ed.). Tropical stream ecology. 1. ed. Amsterdam: Academic Press, 2008. p. 107-146.

ZAR, J. H. Bioestatistical analysis. New Jersey: Prentice Hall, 1996. $662 \mathrm{p}$. 